\title{
Editorial: The Long-Lasting Quest for Nuclear Interactions: The Past, the Present and the Future
}

\author{
Laura E. Marcucci ${ }^{1,2 *}$ \\ ${ }^{1}$ Department of Physics "E. Fermi", University of Pisa, Pisa, Italy, ${ }^{2}$ Sezione di Pisa, Istituto Nazionale di Fisica Nucleare, Pisa, Italy
}

Keywords: Nuclear interactions, two- and three-nucleon interactions, chiral effective field theory, lattice QCD, light nuclei, ab-initio methods

Editorial on the Research Topic

The long-lasting quest for nuclear interactions: the past, the present and the future

As it was stated when this Research Topic was launched, despite many years of intense research, our understanding of the force that holds the atomic nuclei together is still far from complete, and numerous crucial questions are yet to be answered. The theory of nuclear forces has a long and glorious history, as recently summarized in 1. In a nutshell, in the 1990s, meson-theoretic and pure phenomenological approaches were used to describe the nuclear interaction, with models which were able to reproduce the large set of two-nucleon $(2 \mathrm{~N})$ experimental data available at that time (or more properly the phase-shifts arising from a partial wave analysis of these data) with a $\chi^{2} /$ datum close to unity $[2,3]$. However, all these models were relegated in the world of phenomenology and no connection with the underlying theory of Quantum Chromodynamics (QCD) could be found.

At the beginning of the 1990s, we entered in a new still-ongoing phase: nuclear physicists "discovered" QCD and, more importantly, effective field theory (EFT). Following a seminal idea of Weinberg $[4,5]$, several groups developed nuclear interaction models within the so-called chiral EFT ( $\chi \mathrm{EFT})$, an EFT where the spontaneous breaking of QCD chiral symmetry plays a crucial role. The ${ }_{x}$ EFT approach is reviewed and discussed at length in essentially all contributions to this Research Topic, as we will outline below. Due to lack of space, here we only mention that the accuracy nowadays reached by the chiral models for nuclear interactions is comparable to, if not even better than, the one reached by the "old" phenomenological models.

Let us quickly review the different contributions to this Research Topic. Four of the most active groups working to derive nuclear force within the ${ }_{\mathrm{EFT}} \mathrm{Epproach}$ are among the contributors to this Research Topic. In particular, Epelbaum et al. review the most recent achievements of the so-called Bochum group, focusing on a new generation of nuclear chiral forces derived using the recently proposed semi-local regularization method. The authors also describe the ongoing efforts by the Low-Energy Nuclear Physics International Collaboration (LENPIC) toward developing consistent two- and many-body forces, and discuss selected applications. The contribution of Entem et al. is a clear and thorough review of the latest work of the so-called Idaho group, which has developed ${ }_{x}$ EFT-based potentials, from leading order up to next-to-next-to-next-to-next-to leading order (N4LO). The authors present also a discussion of the most recently developed N5LO contributions, and their effects on $2 \mathrm{~N}$ scattering. In the contribution of Ekström we can find a discussion of the importance of careful model calibration and uncertainty quantification of theoretical predictions. To this aim, the author reviews how statistical computing and methods, such as Bayesian inference methods, can be used in conjunction with ab-initio methods for atomic nuclei, in order to construct $x$ EFT-based nuclear potentials. Finally, Piarulli et al. review the efforts made by two different groups in applying the $\chi_{\mathrm{EFT}}$ approach in coordinate space. This allows to derive chiral potential models which are local and suitable to be used in studies of nuclear structure with the 
ab-initio methods based on Quantum Monte Carlo techniques. Selected results, ranging from light nuclei up to ${ }^{16} \mathrm{O}$, as well as neutron matter, are also presented.

Two essential aspects of $\chi$ EFT are also reviewed in the Research Topic. The first one is the problem of renormalization of chiral nuclear forces. In his contribution, Van Kolck summarizes the huge body of work done on this subject and describes alternative approaches which can be used to achieve renormalization order by order. The second essential aspect is the proper quantification of uncertainties on $2 \mathrm{~N}$ phase shifts analysis and, consequently, on the $2 \mathrm{~N}$ interactions. The work done in Granada in determining the $2 \mathrm{~N}$ scattering database is reviewed by Arriola et al., who also discuss the impact of this database on the determination of the $2 \mathrm{~N}$ force.

The ${ }_{x} \mathrm{EFT}$ approach can be suitably adapted to derive also more general baryon-baryon interactions. For instance, Petschauer et al. review the systematic derivation of hyperon-nuclear forces from the symmetries of QCD within non-relativistic SU(3) $\times$ EFT, and also discuss several applications, ranging from hyperon-nucleon scattering up to hyperon-nuclear few- and many-body systems, including hypernuclei and neutron star matter. The contribution of de Vries et al., instead, is about the parity-violating but time-reversal conserving, and the parity-violating and time-reversal-violating forces in nuclei. These forces are a tiny component of the total interaction between nucleons, but their study is extremely interesting, because they allow one to obtain information on fundamental symmetries using nuclear systems. In this contribution, the authors review how the above mentioned interactions are derived in $\chi \mathrm{EFT}$, and discuss the effects of these forces on several few-nucleon observables.

Two papers are devoted to approaches alternative to $\chi$ EFT used to derive nuclear interaction models. In the first one, Fernández et al. review the different quark models used to describe $2 \mathrm{~N}$ and, more in general, baryon-baryon interaction. These models are nonperturbative QCD-inspired and retain quark and gluons as degrees of freedom. Special attention is devoted to the constituent quark model. This model has recently attracted large interest, because its description of the $2 \mathrm{~N}$ interaction at short distances is qualitatively consistent with the lattice-QCD simulations near the physical quark masses. An example of nuclear interaction models derived from lattice-QCD simulations is presented by Aoki et al., who discuss the so-called HAL QCD method, and review the derivation of the central, tensor, and spin-orbit components present in the $2 \mathrm{~N}$ force, and of the three-nucleon $(3 N)$ interaction.

The nuclear interaction models derived in $x \mathrm{EFT}$ reproduce with great accuracy $2 \mathrm{~N}$ bound and scattering states. However, it is essential to know how they perform in reproducing other nuclear systems, starting from $A>2$ light nuclei. In order to address this question, very accurate ab-initio methods are fundamental. In this Research Topic, the latest advances of two of these methods are reviewed: Lazauskas et al. discuss the application of the Faddeev-Yakubovsky equations in configuration space to the

\section{REFERENCES}

1. Machleidt R. Historical perspective and future prospects for nuclear interactions. Int J Mod Phys E (2017) 26(11):1730005. doi:10.1142/ s0218301317300053 four- and five-nucleon systems; Marcucci et al. review the hyperspherical harmonics method and its latest results obtained with both local and non-local chiral potentials for three- and four-body nuclear bound and scattering systems. Together with the method based on the Faddeev-Yakubovsky equations in momentum space $[6,7]$, the presented methods are among the most accurate ones for bound and scattering $A=3,4$ nuclear systems.

In order to use chiral forces in larger nuclei up to nuclear matter, further work is necessary. Some issues related to the use of nuclear forces in the medium are discussed by Sammarruca et al. and Holt et al. In particular, Sammarruca et al. present a review of their recent studies of the equation of state for symmetric nuclear matter and pure neutron matter, using state-of-the-art chiral interactions. Holt et al. discuss the implementation of $3 \mathrm{~N}$ forces in many-body nuclear structure and reaction studies, presenting an approach which employs a medium-dependent $2 \mathrm{~N}$ interaction. The authors also discuss several applications.

We conclude this overview with the contribution of Richard, which is devoted to the physics of low-energy antiprotons and, most importantly in this context, its link with nuclear forces. In fact, a good understanding of antinucleon-nucleon and antinucleonnucleus interactions is necessary in order to investigate important phenomena in astrophysics, as high-energy cosmic rays, or the matter-antimatter asymmetry in early Universe.

In conclusion, this Research Topic has collected the contributions of the researchers most active in the development and understanding of the nuclear interaction and some related topics, as those outlined above. At the end, we hope that this article collection will serve as a useful compendium for practitioners, who everyday apply nuclear potentials in their work and wish to learn about the most significant aspects in an efficient way.

\section{AUTHOR CONTRIBUTIONS}

The author confirms being the sole contributor of this work and approved it for publication.

\section{ACKNOWLEDGMENTS}

The author would like to warmly thank Prof. Ruprecht Machleidt for his deep insights and great support in proposing, managing, and reviewing large part of the editorial work. Last, but not least, the author would like to thank the many colleagues who have enthusiastically answered to the call of submission, and the many reviewers who, with great professionalism, have worked anonymously together with the authors to improve the submitted articles.

2. Machleidt R. High-precision, charge-dependent Bonn nucleon-nucleon potential. Phys Rev C (2001) 63(2):024001. doi:10.1103/PhysRevC.63. 024001

3. Wiringa RB, Stoks VGJ, Schiavilla R. Accurate nucleon-nucleon potential with charge-independence breaking. Phys Rev C (1995) 51(1):38-51. doi:10.1103/ PhysRevC.51.38 
4. Weinberg S. Nuclear forces from chiral Lagrangians. Phys Lett B (1990) 251(2): 288-92. doi:10.1016/0370-2693(90)90938-3

5. Weinberg S. Effective chiral Lagrangians for nucleon-pion interactions and nuclear forces. Nucl Phys B (1991) 363(1):3-18. doi:10.1016/0550-3213(91)90231-L

6. Deltuva A, Fonseca A, Sauer P. Coulomb force effects in few-nucleon systems. Few Body Syst (2019) 60(2):29. doi:10.1007/s00601-019-1496-x

7. Witała H, Golak J, Skibiński R, Topolnicki K, Epelbaum E, Hebeler K, et al. Application of semilocal coordinate-space regularized chiral forces to elastic $\mathrm{Nd}$ scattering and breakup. Few Body Syst (2019) 60(1):19. doi:10.1007/s00601-0191485-0
Conflict of Interest: The author declares that the research was conducted in the absence of any commercial or financial relationships that could be construed as a potential conflict of interest.

Copyright () 2020 Marcucci. This is an open-access article distributed under the terms of the Creative Commons Attribution License (CC BY). The use, distribution or reproduction in other forums is permitted, provided the original author(s) and the copyright owner(s) are credited and that the original publication in this journal is cited, in accordance with accepted academic practice. No use, distribution or reproduction is permitted which does not comply with these terms. 